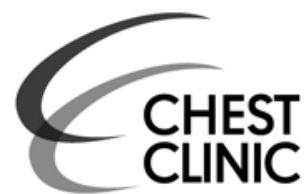

- Additional materials are published online only. To view these files please visit the journal online (http://dx.doi.org/ 10.1136/thoraxjnl-2011200994).

${ }^{1}$ Burton Hospitals NHS Foundation Trust Burton-on-Trent, UK ${ }^{2}$ Department of Respiratory Medicine, Leeds Teaching Hospitals NHS Trust, Leeds, UK ${ }^{3}$ Department of Epidemiology, University of Nottingham,

Nottingham, UK

${ }^{4}$ Department of Respiratory Medicine, North Tees General Hospital, Stockton-on-Tees, UK ${ }^{5}$ Department of Respiratory Medicine, Glenfield Hospital, Leicester, UK

${ }^{6}$ The Information Centre for Health and Social Care, Leeds, UK

${ }^{7}$ Department of Respiratory Medicine, University Hospitals of Birmingham, Birmingham, UK ${ }^{8}$ Department of Respiratory Medicine, Papworth Hospital, Cambridge, UK

${ }^{9}$ Department of Respiratory Medicine, University of Nottingham, Nottingham, UK

\section{Correspondence to} Dr Paul Beckett, Burton Hospitals NHS Foundation Trust, Queens Hospital Belvedere Road, Burton-on-Trent DE13 ORB, UK;

emailpaulbeckett@gmail.com

Received 4 September 2011 Accepted 7 June 2012 Published Online First 6 July 2012

\title{
Clinical management of older people with non-small cell lung cancer in England
}

\author{
Paul Beckett, ${ }^{1}$ Matthew Callister, ${ }^{2}$ Laila J Tata, ${ }^{3}$ Richard Harrison, ${ }^{4}$ \\ Michael D Peake, ${ }^{5}$ Roz Stanley, ${ }^{6}$ lan Woolhouse, ${ }^{7}$ Mark Slade, ${ }^{8}$ Richard B Hubbard ${ }^{9}$
}

\section{ABSTRACT}

Data for 25261 patients with non-small cell lung cancer whose details were submitted to the National Lung Cancer Audit in England were analysed to assess the effect of age at diagnosis on their clinical management, after accounting for sex, stage, performance status and comorbidity. Multivariate logistic regression showed the odds of having histocytological confirmation and anticancer treatment decreased progressively with age, and was also lower in women. It is likely that these results have a multifactorial explanation, and further research into the attitudes of patients, carers and healthcare professionals, and clinical trials of treatment in older populations, are necessary.

\section{INTRODUCTION}

Approximately 40000 new cases of lung cancer are diagnosed in England and Wales each year, making it the second commonest cancer in men (after prostate) and women (after breast) ${ }^{1}$ and the commonest cause of cancer-related death in both sexes. Treatment and survival rates vary across the UK and do not match those achieved in comparable healthcare systems. Despite recent scrutiny of primary care to try to explain these observations, ${ }^{2}$ it is possible that lower levels of investigation and treatment in older patients in secondary care may explain some of the variation observed.

The National Lung Cancer Audit (NLCA) in the UK is commissioned by the Healthcare Quality Improvement Partnership with funds from the Department of Health and maintained jointly by the Health and Social Care Information Centre and the Royal College of Physicians. It has collected data of progressively improving completeness since 2005. These are now of sufficient quality to permit analysis of factors relating to treatment and outcome. $^{3}$ We have used NLCA data to quantify the association between age and the presence of histocytological confirmation of diagnosis and/or receipt of anticancer treatment (surgery, chemotherapy or radiotherapy) in people with non-small cell lung cancer (NSCLC).

\section{METHODS}

We obtained data for the 30098 people with lung cancer first seen in England in 2009 submitted to the NLCA. A comparison of these cases with published lung cancer registrations from the Office of National Statistics for England ${ }^{1}$ shows that the
NLCA ascertained the vast majority of lung cancers and case distribution by age at diagnosis was representative of national registry data (see online supplementary figure 1). Of all cases, we excluded those with pathologically confirmed small cell lung cancer or confirmed/suspected mesothelioma, leaving 25261 people with histocytologically confirmed or clinically diagnosed NSCLC.

The clinical management measures were patients' receipt of histocytological confirmation of NSCLC and receipt of any anticancer treatment (recorded treatment with surgery, radiotherapy or chemotherapy). We assessed these by age at diagnosis grouped into 10-year age bands (60-69 year olds were used as the reference group in analyses), sex, stage (as classified by the American Joint Committee on Cancer and Union Internationale Contre le Cancer V.6), performance status (as classified by the Eastern Cooperative Oncology Group) and recording of a significant diagnosed comorbidity.

We used multivariate logistic regression to quantify the association of age with our clinical management outcomes, adjusting for sex and the other important clinical features. Receipt of presence of histocytological confirmation was also included as a covariate in the multivariable model for anticancer treatment. Finally we looked for evidence of interaction between age and sex by fitting a multiplicative interaction term.

All statistical analyses were carried out using Stata V.SE11.

\section{RESULTS}

The 25261 patients with NSCLC analysed had a median age of 72 years (interquartile range 64-80). Demographics and clinical features of the patients according to age and the clinical management outcomes are shown in online supplementary tables 1 and 2. Overall, 68\% of patients had histocytological confirmation of the diagnosis and $57 \%$ received anticancer treatment.

Table 1 shows the ORs for histocytological confirmation by age and sex adjusted for clinical features. Other than for patients in the youngest age group ( $<40$ years), the multivariable model showed the odds of histocytological confirmation decreased progressively with age. For example, compared with patients aged 60-69 years, the OR was 1.4 for patients aged $40-49$ and 0.36 for those aged 80-89. Female sex (OR 0.81 compared with men) and having at least one recorded comorbidity (OR 0.75 ) were associated with reduced odds of 
histocytological confirmation, which also decreased markedly with worsening performance status, whereas the relationship with stage was n-shaped with the largest odds being in those with stage IIIB cancer.

We found a statistically significant interaction between age and sex in our multivariate model (likelihood ratio test $\mathrm{p}=0.0009$ ) which showed that, although similar between men and women under 60 years of age, women were less likely than men to receive histocytological confirmation as they became older. For example, the OR was 1.03 (95\% CI 0.66 to 1.60) for women compared with men aged $40-49$ and the OR was 0.72 (95\% CI 0.65 to 0.81 ) for patients aged $80-89$ (figure 1).

For anticancer treatment, the results were very similar to those for histocytological confirmation (table 1) with the odds of anticancer treatment decreasing progressively with age. For example, compared with patients aged 60-69 years, the OR was 1.97 for patients aged 40-49 and 0.24 for those aged 80-89. Female sex (OR 0.91 compared with men) and having at least one recorded comorbidity ( $\mathrm{OR}$ 0.75) were associated with reduced odds of anticancer treatment, which also decreased markedly with worsening performance status and disease stage. Again there was evidence of an interaction between age and sex (likelihood ratio test $\mathrm{p}=0.007$ ), for example, the OR was 0.97 (95\% CI 0.62 to 1.51$)$ for women compared with men aged 40-49 and the OR was 0.78 (95\% CI 0.69 to 0.89 ) for patients aged 80-89 (figure 1).

\section{CONCLUSIONS}

Our results suggest that age has an important impact on the investigation and treatment of people with lung cancer in England. After adjusting for the effects of sex, stage, perfor- mance status and comorbidity there was a progressive decline in patients' receipt of histocytological confirmation and anticancer treatment from age 50 years onwards. We also found that women with lung cancer were 19\% less likely to have histocytological confirmation of their cancer than men and $9 \%$ less likely to have anticancer treatment after adjusting for important clinical features. These sex differences were mostly in patients aged 60-89 years, despite very similar proportions of lung cancers in men and women across these age groups.

The main strengths of our study are the large size of the NLCA datasets, which has been shown to be representative of people with lung cancer in the UK. ${ }^{3}$ NLCA data completeness has progressively improved since its inception in 2005, with the result that the 30098 patients analysed for this study represent almost $100 \%$ of the cases managed in secondary care that would be expected from population incidence figures. Furthermore comparison between our dataset and the most recently published data on cancer registrations for England does not suggest that older patients are under-represented in the audit dataset, a finding that supports more detailed previously published research. ${ }^{3}$ The main weakness of our study is the lack of robust data on comorbidity routinely available in the NLCA, although information on stage and performance status are robust and more complete than other routine sources.

Our results are in line with earlier pilot research on a smaller cohort of 1652 patients from 48 English hospitals with all types of lung cancer which found significant inverse correlations between age and histocytological diagnosis, anticancer treatment and survival. ${ }^{4}$ Histocytological confirmation was obtained in $89 \%$ of their patients under age 65 years and $80 \%$ in those over 75 years and anticancer treatment was given in $78 \%$

Table 1 ORs for histocytological confirmation and anticancer treatment

\begin{tabular}{|c|c|c|c|c|c|c|}
\hline & \multicolumn{3}{|c|}{ Histocytological confirmation } & \multicolumn{3}{|c|}{ Anticancer treatment } \\
\hline & $\mathbf{O R}^{*}$ & $95 \% \mathrm{Cl}$ & p Value & $\mathbf{O R}^{*}$ & $95 \% \mathrm{Cl}$ & p Value \\
\hline Male & 1 & - & - & 1 & - & - \\
\hline Female & 0.81 & 0.77 to 0.86 & $<0.001$ & 0.91 & 0.86 to 0.96 & 0.002 \\
\hline \multicolumn{7}{|l|}{ Age (years) } \\
\hline$<40$ & 0.85 & 0.55 to 1.31 & 0.451 & 1.10 & 0.70 to 1.72 & 0.671 \\
\hline $40-49$ & 1.40 & 1.12 to 1.76 & 0.004 & 1.97 & 1.57 to 2.47 & $<0.001$ \\
\hline $50-59$ & 1.34 & 1.19 to 1.52 & $<0.001$ & 1.40 & 1.25 to 1.58 & $<0.001$ \\
\hline $70-79$ & 0.71 & 0.65 to 0.76 & $<0.001$ & 0.59 & 0.55 to 0.63 & $<0.001$ \\
\hline $80-89$ & 0.36 & 0.33 to 0.39 & $<0.001$ & 0.24 & 0.22 to 0.26 & $<0.001$ \\
\hline$>90$ & 0.12 & 0.10 to 0.15 & $<0.001$ & 0.07 & 0.05 to 0.10 & $<0.001$ \\
\hline \multicolumn{7}{|l|}{ Cancer stage } \\
\hline IA & 1 & - & - & 1 & - & - \\
\hline IB & 1.47 & 1.23 to 1.75 & $<0.001$ & 1.29 & 1.07 to 1.56 & 0.007 \\
\hline IV & 1.38 & 1.20 to 1.59 & $<0.001$ & 0.59 & 0.51 to 0.68 & $<0.001$ \\
\hline Unknown & 0.87 & 0.75 to 1.00 & 0.055 & 0.52 & 0.44 to 0.60 & $<0.001$ \\
\hline \multicolumn{7}{|l|}{ Performance status } \\
\hline 0 & 1 & - & - & 1 & - & - \\
\hline 1 & 0.93 & 0.83 to 1.04 & 0.205 & 0.84 & 0.76 to 0.94 & 0.002 \\
\hline 2 & 0.47 & 0.42 to 0.53 & $<0.001$ & 0.34 & 0.30 to 0.38 & $<0.001$ \\
\hline 3 & 0.20 & 0.18 to 0.23 & $<0.001$ & 0.10 & 0.09 to 0.11 & $<0.001$ \\
\hline 4 & 0.10 & 0.09 to 0.12 & $<0.001$ & 0.03 & 0.02 to 0.04 & $<0.001$ \\
\hline Unknown & 0.43 & 0.38 to 0.48 & $<0.001$ & 0.31 & 0.28 to 0.34 & $<0.001$ \\
\hline Diagnosed comorbidity & 0.75 & 0.69 to 0.82 & $<0.001$ & 0.75 & 0.69 to 0.82 & $<0.001$ \\
\hline Histocytologically confirmed & - & - & - & 4.02 & 3.75 to 4.30 & $<0.001$ \\
\hline
\end{tabular}

\footnotetext{
*Fully adjusted for all variables in the table.
} 
Figure 1 Odds ratios for histocytological confirmation $(A)$ and anti-cancer treatment $(B)$ in women compared with men (reference group), stratified by age group.

A

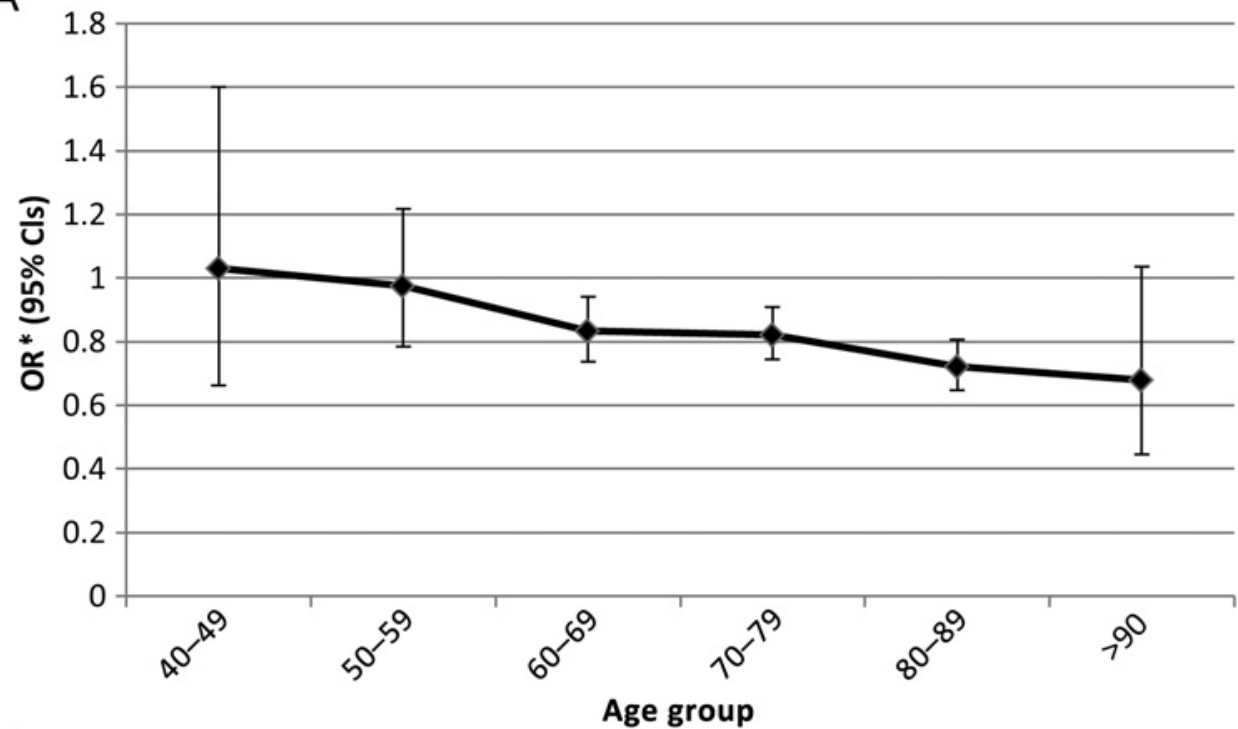

B

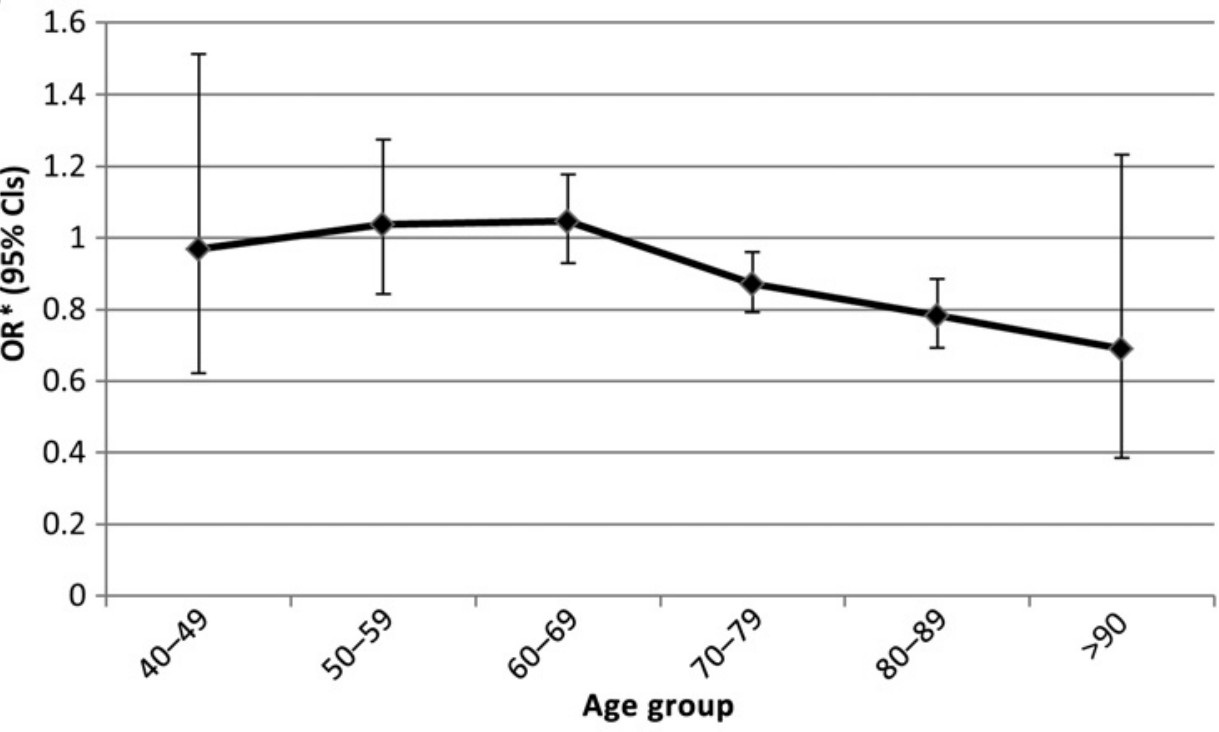

*ORs adjusted for stage, performance status, comorbidity (and histocytological confirmation in the anti-cancer treatment model only)

and $49 \%$ respectively. These are slightly higher confirmation and treatment proportions than in NLCA cases overall, however these 1652 patients were a more selected population by virtue of all undergoing bronchoscopy. Similar results have been obtained from studies carried out in the USA, Canada and Europe.

Although there is considerable evidence that tumours behave differently, and outcomes differ, in men and women, there is very little published research on the effect of gender on disease management in patients with lung cancer, since most research has adjusted for sex rather than examining its effect. It is difficult to find a satisfactory explanation for the results observed in our analyses. It is possible that men and women perceive the benefits of a histocytological diagnosis and anticancer treatment differently or that healthcare professionals have a different attitude to a more aggressive management approach in women.

There is good evidence that older patients with comparable clinical features to younger patients can tolerate and benefit equally from investigation and treatment. A recent European
Organisation for Research and Treatment of Cancer statement on treatment in older people, based on a detailed literature review and expert consensus, acknowledges the lack of research evidence to guide clinical decisions about treatment but also clearly states that suitability for treatment should not be defined upon chronological age alone. ${ }^{5}$

How are patients and lung cancer multidisciplinary teams to interpret these results? It is likely that the less active approach to management of lung cancer observed in older people has a multifactorial explanation. Difficulty of access to services, therapeutic nihilism in primary and secondary care, overestimation of the risks of treatment-related toxicity, and illinformed patient choice, based perhaps upon the experiences of friends or relatives undergoing different treatment for different cancers, may all play a part. However, it is the multidisciplinary teams who provide key decisions and it is incumbent upon them to examine more closely their own results through local audit and to consider whether their investigation and treatment protocols may inadvertently discriminate against older patients. Further research into attitudes of patients, carers and healthcare 
professionals, and clinical trials of treatment in older populations are vital if we are to ensure that all patients receive the very best care, regardless of their age.

Contributors PB, RS, MDP and IW lead the team responsible for audit data. RH provided the initial idea for the analysis. PB analysed the data under the supervision of RBH and LJT. PB wrote the paper with substantial input from all authors on content, style and presentation.

Funding The National Lung Cancer Audit in the UK is commissioned by the Healthcare Quality Improvement Partnership (HOIP) with funds from the Department of Health and maintained jointly by the Health and Social Care Information Centre (HSCIC) and the Royal College of Physicians.

Competing interests None.

Provenance and peer review Not commissioned; externally peer reviewed.

\section{REFERENCES}

1. Office for National Statistics. Cancer Statistics Registration, England, Series MB1. 2009. http://www.ons.gov.uk/ons/rel/vsob1/cancer-statistics-

registrations-england-series-mb1-/no-40-2009/cancer-registrations-2009.xls (accessed 21 Feb 2012).

2. Holmberg L, Sandin F, Bray F, et al. National comparisons of lung cancer survival in England, Norway and Sweden 2001-2004: differences occur early in follow-up. Thorax 2010;65:436-41.

3. Rich AL, Tata LJ, Stanley RA, et al. Lung cancer in England: information from the National Lung Cancer Audit (LUCADA). Lung cancer 2011;72:16-22.

4. Peake MD, Thompson S, Lowe D, et al. Ageism in the management of lung cancer Age and Ageing 2003;32:171-7.

5. Pallis AG, Gridelli C, van Meerbeeck JP, et al. EORTC Elderly Task Force and Lung Cancer Group and International Society for Geriatric Oncology (SIOG) experts' opinion for the treatment of non-small-cell lung cancer in an elderly population. Ann Oncol 2010:21:692-706

\section{Journal club}

\section{CPAP may reduce cardiovascular mortality in patients with OSA}

This double-blind randomised crossover study of patients with moderate-to-severe obstructive sleep apnoea (OSA) compared 3 months of therapeutic continuous positive airway pressure (CPAP) with 3 months of sham CPAP with a 1 month wash-out period in between. Markers of the metabolic syndrome were measured before and after each CPAP treatment.

The study population comprised 86 CPAP naïve patients aged 30-65 who were recruited from a sleep laboratory in New Delhi, India. Exclusion criteria were previous or current treatment for hypertension, diabetes, dyslipidemia or any evidence of end-organ damage due to these conditions.

There were modest, statistically significant, reductions in systolic and diastolic blood pressure, glycated haemoglobin, triglycerides, low-density lipoprotein, non-high-density lipoprotein and total cholesterol after CPAP therapy as compared with sham therapy. Additionally, there were significant decreases in body mass index and visceral and subcutaneous fat. Carotid intimal thickness and insulin resistance did not differ significantly. Applying the authors' criteria for the metabolic syndrome, there was a net reduction in the number of patients affected.

Predefined subgroup analysis of patients whose mean adherence with CPAP was five or more hours per night $(n=51)$ revealed, compared with the whole population, significantly greater reductions in systolic and diastolic blood pressure, glycated haemoglobin, triglyceride, $\mathrm{LDH}$ and total cholesterol.

The study uses surrogate end points for cardiovascular mortality. CPAP reduces cardiovascular mortality in patients with OSA and CPAP is the recommended treatment for patients with moderate-to-severe OSA.

Further research is needed before we can conclude that additional cardiovascular mortality reduction benefit accrues to this subset of patients with moderate OSA who have the metabolic syndrome.

- Sharma SK, Agrawal S, Damodaran D, et al. CPAP for the metabolic syndrome in patients with obstructive sleep apnoea. N Engl J Med 2011;365:2277-86.

\section{Carl J Reynolds}

Correspondence to Dr Carl J Reynolds, CT1, Chelsea and Westminster NHS Trust, Department of Acute Medicine, 369 Fulham Road, London SW10 9NH, UK; carl.reynolds@nhs.net

Published Online First 10 February 2012

Thorax 2012;67:839. doi:10.1136/thoraxjnl-2012-201669 